\title{
Elucidation of the molecular mechanisms of anaplastic thyroid carcinoma by integrated miRNA and mRNA analysis
}

\author{
GUOPING LIU*, KAINAN WU* and YUAN SHENG \\ Department of Breast Surgery, Changhai Hospital, Second Military Medical University, Shanghai 200433, P.R. China
}

Received January 28, 2016; Accepted March 24, 2016

DOI: $10.3892 /$ or.2016.5064

\begin{abstract}
To elucidate the complex molecular mechanisms of anaplastic thyroid carcinoma (ATC), the mRNA and miRNA expression profiles of ATC were systematically explored. A total of 55 common differentially expressed genes (DEGs) were obtained from two mRNA expression datasets including 23 ATC samples and 24 paired normal samples. Gene expression levels of three randomly selected DEGs, VCAN, COL5A1 and KCNJ16, were examined using RT-PCR in 10 ATC samples. Notably, the ATC and normal samples were clearly classified into two groups based on their common DEGs. Moreover 23 common DEGs, such as TG, NKX2-1, KCNJ16 and $C T H R C 1$, were predicted to be the potential targets of 17 identified miRNAs in ATC. Meanwhile, several miRNA target genes were associated with biological processes related to tumor progression such as angiogenesis, cell migration or growth and potassium channel regulation. In summary, the poor prognosis of ATC is possibly caused via complex biological processes. Firstly, angiogenesis was activated by the high expression of CTHRC1, VCAN and POSTN, providing necessary nutrition for tumor cells. Then tumor distant metastasis was induced via stimulation of cell migration and cell growth or regulation of cell-cell interaction. Moreover, intracellular potassium concentration changes promoted ATC progression indirectly. Hence, identification of these critical DEGs was valuable in understanding the molecular mechanisms of ATC.
\end{abstract}

\section{Introduction}

The incidence of thyroid cancer, one of the most common endocrine malignancies, has increased rapidly in recent years based on worldwide statistics (1). The annual number of newly diagnosed thyroid cancer cases is $129 / 1,000,000$, and the associated deaths are 5/1,000,000 (2). Thyroid cancer can be divided into well-differentiated carcinomas (such as papil-

Correspondence to: Dr Yuan Sheng, Department of Breast Surgery, Changhai Hospital, Second Military Medical University, 168 Changhai Road, Shanghai 200433, P.R. China

E-mail: sheng_yuan_2015@163.com

${ }^{*}$ Contributed equally

Key words: anaplastic thyroid carcinomas, microarray analysis, differentially expressed genes, miRNA, RT-PCR lary or follicular carcinomas) and anaplastic thyroid cancer (ATC) (3). The percentage of ATC is low, ranging from 1 to 5\% of all thyroid cancers, but accounts for $\sim 14-50 \%$ of deaths (4). The poor prognosis and the high rate of distant metastases of ATC lead to a 5-year survival rate of $7 \%$ (5).

Considering the complexity of the molecular mechanisms, various studies have been carried out such as mRNA (6) and miRNA expression profiling (5) by microarray, gene mutation whole exome sequencing (7), cytogenetic analysis and comparative genomic hybridization $(\mathrm{CGH})$ microarray (8). Several de novo mutations including TP53, $\beta$-catenin and PIK3CA have been identified in ATC whereas in pre-existing mutations in PTC (papillary thyroid cancer) mutations such as $R A S$ and $B R A F$ have been found (4). mRNA and miRNA expression levels have also been demonstrated to be critical in tumor progression. A study by von Roemeling et al showed that stearoyl-CoA desaturase 1 (SCDI) associated with fatty acid metabolism is highly expressed in ATC compared with that in normal samples (9). Gene expression analysis of ATC and PTC demonstrated that most of the DEGs were common in both, but ATC contained more genes associated with epithelial to mesenchymal transition (EMT), dedifferentiation and glycolytic phenotypes (6). Furthermore, miRNA expression profile analysis of 11 ATC samples showed that 17 common miRNAs were downregulated and one was upregulated (5).

Although previous research has been carried out for ATC, the combination analysis of mRNA and miRNA expression profiles has not yet been systematically explored. A substantial amount of mRNA expression datasets have been submitted to the GEO (Gene Expression Omnibus) database and bioinformatic methods have been demonstrated to be valuable for molecular mechanism investigation (10). In the present study, mRNA expression datasets from different laboratories were analyzed and several DEGs were identified by RT-PCR. Then common DEGs were subjected to subsequent function analysis. Furthermore, miRNA and mRNA expression levels were integrated for the elucidation of the molecular mechanisms.

\section{Materials and methods}

Gene expression profiles. Two gene expression profiles GSE33630 and GSE65144 submitted by Dom et al (11) and von Roemeling et al (9), respectively, were downloaded from the Gene Expression Omnibus (GEO, http://www.ncbi.nlm. nih.gov/geo/). For the GSE33630 dataset, 11 ATC samples 

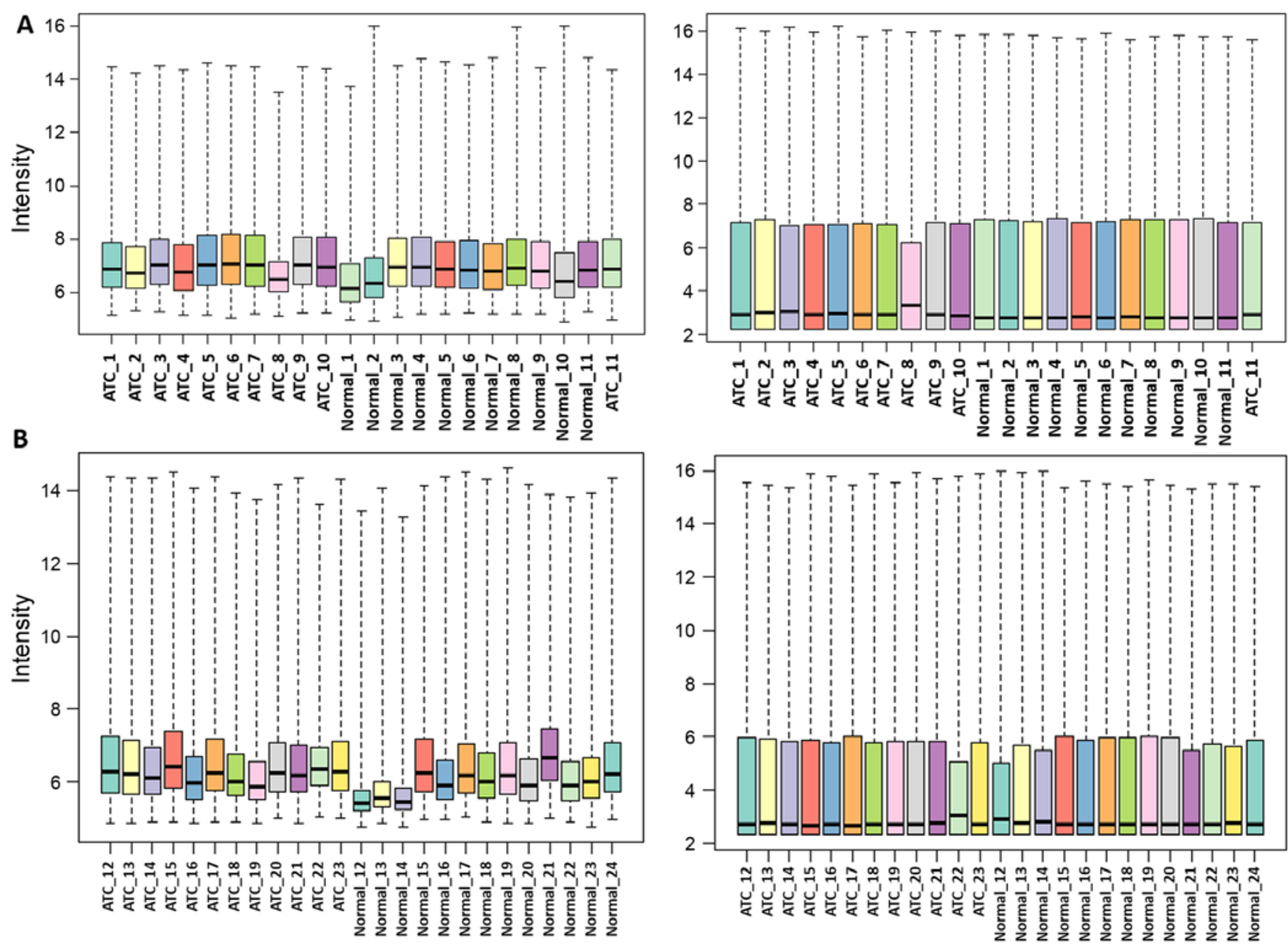

Figure 1. Box plots of gene expression for paired tumor and normal thyroid tissues before normalization (left) and after normalization (right). (A) GSE33630 and (B) GSE65144.

and 11 paired normal samples were selected for subsequent analysis. The GSE65144 dataset consisted of 12 ATC samples and 13 normal samples (12 matched and 1 unmatched). Experiments for the two datasets were carried out using GPL570 platform (HG-U133_Plus_2, Affymetrix Human Genome U133 Plus 2.0 array).

Data preprocessing and screening of DEGs. The mRNA expression profiles were subjected to $\log 2$ transformation, background correction and normalization using the GeneChip Robust Multi-array Analysis (GCRMA) method within the Bioconductor package (http://www.bioconductor.org) (12). The uninformative probe sets such as control probe sets, and genes with low expression variance were filtered out. For genes with multiple probes, the average expression value was calculated. Finally, the significant DEGs were identified using the Limma (Linear Models for Microarray Analysis) (13) package with criteria of adjustment of $p<0.01$ and $\log 2$ fold change (FC) $\mid \geq 2$. DEGs with a fold change $>5$ were used for downstream analysis. The heat map was constructed using the heat map method in Bioconductor.

Quantitative RT-PCR analysis. The mRNA expression levels of three randomly selected DEGs, VCAN, COL5Al and
KCNJ16, were examined using RT-PCR. The total RNA was extracted from the 10 ATC samples and the adjacent normal tissues using TRIzol reagent (Thermo Fisher Scientific, Inc., USA) according to the manufacturer's instructions. cDNA was obtained using M-MLV reverse transcriptase based on the manufacturer's protocol (Promega, Madison, WI, USA). The VCAN, COL5A1 and KCNJ16 mRNA expression levels were detected using 7500 Real-Time PCR system (Thermo Fisher Scientific). Relative quantification was normalized using GAPDH mRNA expression and calculated with the $2^{-\Delta \mathrm{Ct}}$ method. The primer sequence list is as follows: $V C A N$ forward, 5'-CACAACCCGCATTTGAACTTG-3' and reverse, 5'-CGC ACGCCTGGAGTTCTT-3'; COL5A1 forward, 5'-ACAACTT GCCTGATGGAA TAACAA-3' and reverse, 5'-CCGGGCCT TTGGAAGATC-3'; KCNJ16 forward, 5'-TCAATGCGGACG CAAAATAC-3' and reverse, 5'-AATCGTCTTCTTGCTCTT CTCTTCTC-3'; and GAPDH forward, 5'-TGACTTCAACAG CGACACCCA-3' and reverse, 5'-CACCCTGTTGCTGTAGC CAAA-3'.

Functional and pathway enrichment analysis. In order to explore the biological processes involved in ATC, functional and pathway enrichment analyses for the common DEGs were carried out using Database for Annotation, Visualization and 


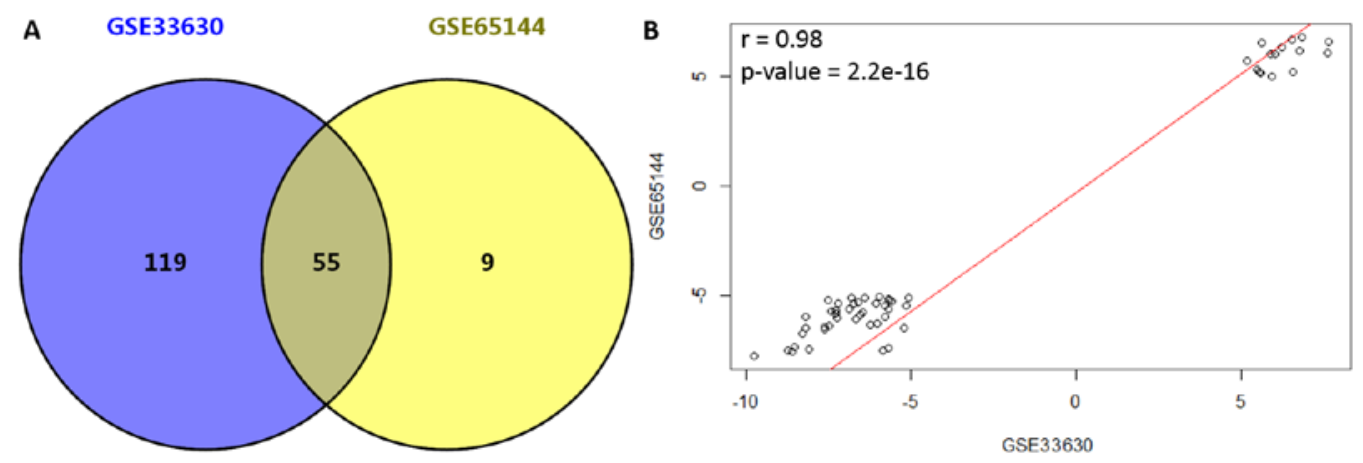

Figure 2. (A) Venn diagram of the differentially expressed genes (DEGs) that were screened. (B) Expression value correlation for the common DEGs in GSE33630 and GSE65144.
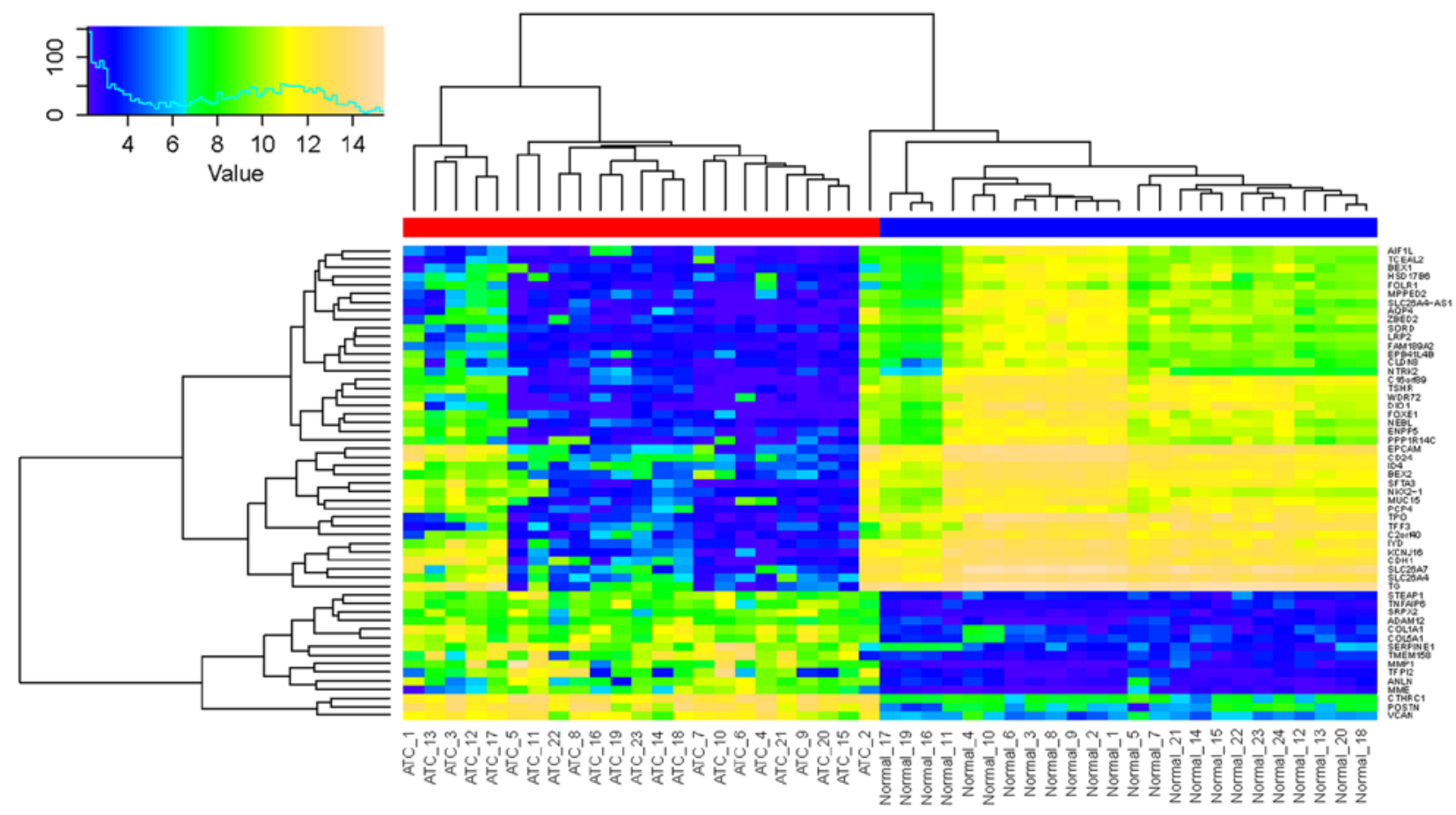

Figure 3. Heat map of the common differentially expressed genes (DEGs). The yellow color represents higher expression and the blue color represents lower expression. The red and blue bars on the top represent ATC and normal samples, respectively.

Integrated Discovery (DAVID) online tools (14), which is based on the Gene Ontology (GO) (15) and Kyoto Encyclopedia of Genes and Genomes (KEGG) (16) databases. The criterion for significantly enriched pathways was set as $\mathrm{p} \leq 0.05$. GO terms, consisting of biological processes (BP), cellular components (CC) and molecular functions (MF), were screened with a $\mathrm{p} \leq 0.05$.

Gene interaction and miRNA regulation network analysis. The physical interaction and validated pathway interaction network for the common DEGs was constructed using the Gene Multiple Association Network Integration Algorithm (GeneMANIA, http://www.genemania.org/) (17). Moreover, 17 deregulated miRNAs from the study of Hebrant et al (5) were integrated for the exploration of miRNA-mRNA interaction by using the CyTargetLinker plugin of Cytoscape (18).

\section{Results}

Common DEGs. After background correction and normalization, the medians of the gene expression values were almost at the same level indicating that the data were suitable for subsequent analysis (Fig. 1). DEGs for the two datasets were identified independently, and a total of 174 and 64 DEGs were screened out for GSE33630 and GSE65144, respectively. Moreover, 55 DEGs (accounting for 32 and 85\% for GSE33630 and GSE65144, respectively) were found to be simultaneously differentially expressed in these two datasets (Fig. 2A). Among the common DEGs, 15 genes were upregulated and 40 were downregulated (data not shown). In addition, correlation of expression values for the 55 common DEGs was 0.98 (p<2.2e-16, Fig. 2B).

Furthermore, the 55 common DEGs were used for the classification of ATC and normal samples. As indicated in Fig. 3, 
Table I. Relative expression values of $V C A N, C O L 5 A 1$ and $K C N J 16$ in 10 paired tumor and adjacent normal tissues.

\begin{tabular}{|c|c|c|c|c|c|c|}
\hline \multirow[b]{2}{*}{ Sample } & \multicolumn{2}{|c|}{$V C A N\left(2^{-\Delta \mathrm{Ct}}\right)$} & \multicolumn{2}{|c|}{ COL5Al $\left(2^{-\Delta \mathrm{Ct}}\right)$} & \multicolumn{2}{|c|}{$\operatorname{KCNJ16}\left(2^{-\Delta \mathrm{Ct}}\right)$} \\
\hline & Tumor & Normal & Tumor & Normal & Tumor & Normal \\
\hline 1 & 0.3647021 & 0.1466735 & 0.1601689 & 0.0177641 & 0.0101683 & 0.145485 \\
\hline 2 & 0.0286243 & 0.0132945 & 0.216439 & 0.0340146 & 0.0031314 & 0.0238292 \\
\hline 3 & 0.0162446 & 0.0018798 & 0.0059821 & 0.0012272 & 0.0218826 & 0.1202726 \\
\hline 4 & 0.039647 & 0.0060125 & 0.2266865 & 0.0599975 & 0.1942628 & 0.3765939 \\
\hline 5 & 0.5845333 & 0.1055037 & 0.2854041 & 0.0404317 & 0.0180416 & 0.0442845 \\
\hline 6 & 1.6831185 & 0.4974194 & 0.6257863 & 0.0625064 & 0.2327167 & 3.1607958 \\
\hline 7 & 0.962266 & 0.1256899 & 0.0009169 & 0.0003568 & 0.1135657 & 0.2204115 \\
\hline 8 & 1.4817526 & 0.0848966 & 0.003156 & 0.0010707 & 0.0332327 & 0.0556471 \\
\hline 9 & 0.1130402 & 0.0329317 & 0.1000809 & 0.0302228 & 0.0117418 & 0.0237591 \\
\hline 10 & 0.7113506 & 0.2759241 & 0.1035169 & 0.0460322 & 0.0009712 & 0.0465934 \\
\hline Mean & 0.5985279 & 0.1290226 & 0.1728138 & 0.0293624 & 0.0639715 & 0.4217672 \\
\hline SD & 0.6117568 & 0.1542157 & 0.1882915 & 0.0236354 & 0.085654 & 0.9687421 \\
\hline
\end{tabular}
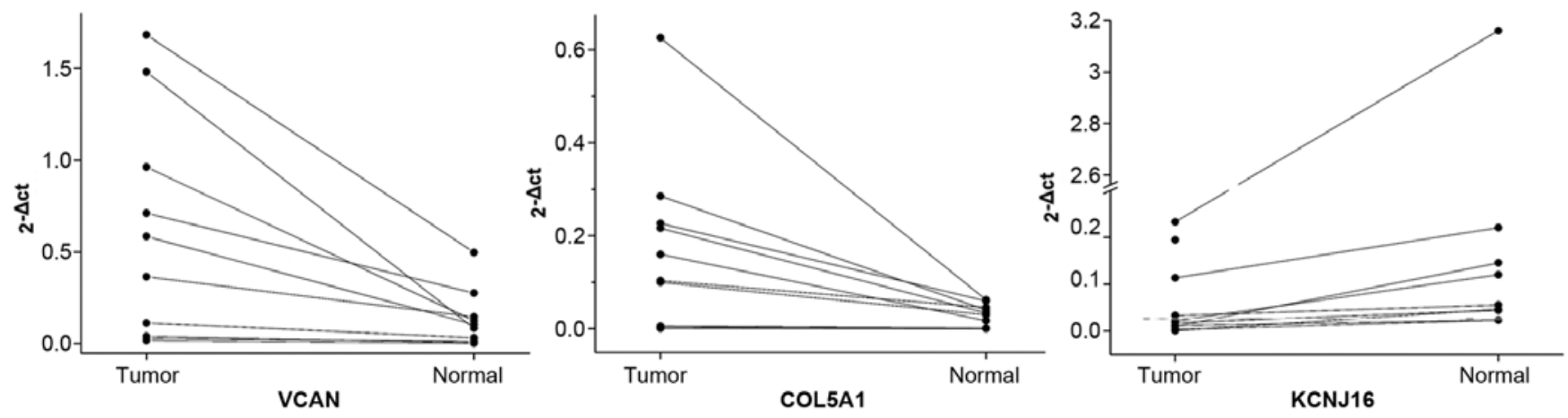

Figure 4. Relative mRNA expression values for $V C A N, C O L 5 A 1$ and $K C N J 16$ in 10 paired tumor and adjacent normal tissues. The $\mathrm{y}$-axis is $2^{-\triangle \mathrm{Ct}}$ and the lines represent tumor and normal tissue pairs.

the ATC and normal samples were clearly classified into two groups except for sample ATC_2, which was possibly due to the smaller expression value variation, ranging from 8-12.

mRNA expression validation by RT-PCR. To verify mRNA expression levels of the identified DEGs, RT-PCR experiments were carried out for three randomly selected genes including VCAN, COL5A1 and KCNJ16. The mRNA expression levels in the 10 ATC samples and adjacent normal tissues were analyzed. Results showed that the expression levels of VCAN and $C O L 5 A 1$ were higher in the tumor tissues than the levels in the adjacent normal tissues; whereas, the mRNA expression level of KCNJ16 was lower in the tumor tissues (Table I and Fig. 4). These results were nearly consistent with those in the microarray analysis.

Functional and pathway enrichment analysis. In order to explore the functions of these common DEGs, functional and pathway enrichment were carried out. The results indicated that the two pathways were significantly enriched for the common DEGs (Table II) and that TG, TPO and $T S H R$ participated in the autoimmune thyroid disease pathway $(\mathrm{p}=0.0083)$, and $C L D N 8, C D H 1$ and $V C A N$ were significantly involved in the cell adhesion molecule (CAM) pathway $(\mathrm{p}=0.049)$.

Futhermore, the common DEGs were mainly related to the cellular components of the extracellular region $(p=2.5 \mathrm{E}$ $05)$ and the proteinaceous extracellular matrix $(\mathrm{p}=7.3 \mathrm{E}-05)$. Some common DEGs, such as TNFAIP6, VCAN, POSTN and $C O L 5 A 1$, were significantly enriched in the carbohydrate binding activity $(\mathrm{p}=0.002)$ and glycosaminoglycan binding function $(p=0.006)$. In addition, hormone regulation-related processes such as regulation of hormone levels $(p=4.6 \mathrm{E}-05)$, hormone biosynthetic processes $(\mathrm{p}=7.3 \mathrm{E}-05)$ and thyroid hormone generation $(\mathrm{p}=1.0 \mathrm{E}-04)$ biological processes were also enriched.

Gene interactionand miRNA regulation analysis. Furthermore, the interaction between these common DEGs was explored to elucidate the potential regulatory mechanism. The results indicated that several important subnetworks were formed based on different pathways. Fig. $5 \mathrm{~A}$ shows that $L R P 2$ can interact with SERPINE and TG physically and TSHR can interact with $T S H B$ physically. Also these common DEGs participated in the autoimmune thyroid disease pathway similar to our results from the pathway enrichment analysis. In addition, $C D H 1$, 
Table II. The enriched pathways for the common differentially expressed genes.

\begin{tabular}{llcll}
\hline Pathway ID & \multicolumn{1}{c}{ Pathway name } & Count & \multicolumn{1}{c}{ Genes } & P-value \\
\hline hsa05320 & Autoimmune thyroid disease & 3 & $T G, T P O$, TSHR & 0.0083 \\
hsa04514 & Cell adhesion molecules (CAMs) & 3 & CLDN8, CDH1, VCAN & 0.049 \\
\hline
\end{tabular}

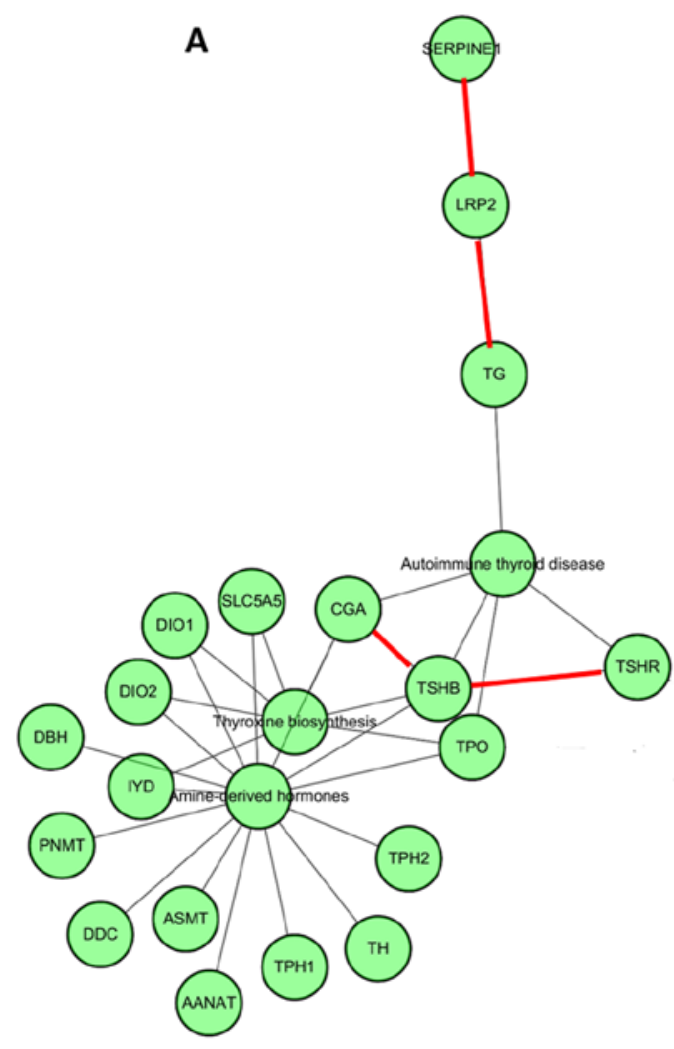

B

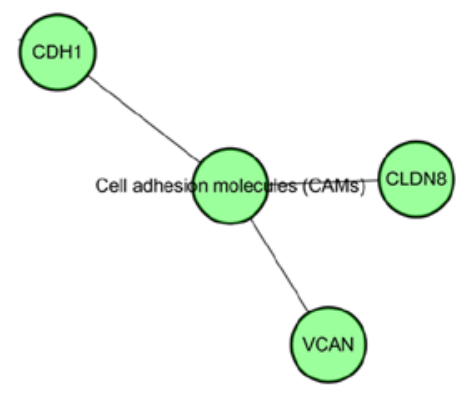

C

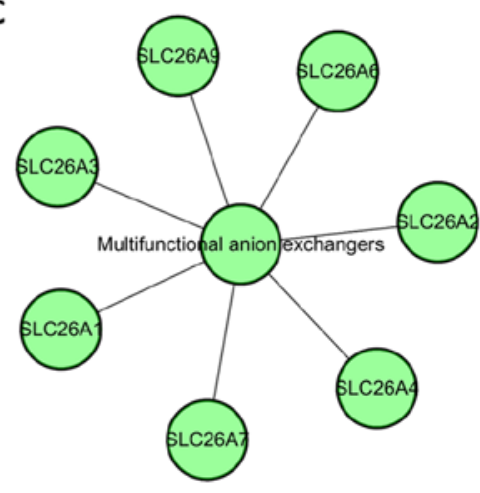

Figure 5. The constructed gene-gene interaction network. (A) The autoimmune thyroid disease subnetwork. (B) The cell adhesion molecule (CAM) subnetwork. (C) The multifunctional anion exchanger subnetwork. The red lines represent physical interaction and the gray lines represent validated pathway interaction.

CLDN8 and VCAN were found to independently participate in cell adhesion molecules. The SLC26A gene family was significantly involved in the multifunctional anion exchanger pathway (Fig. 5B).

For the discovery of miRNAs that can regulate the expression of these common DEGs, the potential targets of 17 reported miRNAs (5) in ATC were predicted. The results showed that 870 and 13,841 target genes were found in the miRTarBase (version 4.4) (19) and TargetScan (version 6.2) (http://www. targetscan.org/) databases, respectively. Among these target genes, 23 genes were identified among the 55 common DEGs, and the miRNA and mRNA interaction network between them is shown in Fig. 6. The network indicated that hsa-let-7f-5p miRNA regulates $T G$ and $N K X 2-1$, and hsa-miR-148b-3p miRNA regulates $K C N J 16$ and $C T H R C 1$ which was validated by our experiments. Other interactions were predicted based on the TargetScan database.

\section{Discussion}

ATC is a type of thyroid cancer with poor prognosis and has been widely studied in mRNA expression, miRNA expres- sion or genome mutational landscape levels. However, efforts trying to elucidate the molecular mechanisms of ATC with the combination of different omics data are rare. In this study, mRNA and miRNA expression levels were combined to explore the potential molecular mechanisms. The mRNA expression analysis showed that 55 common DEGs were simultaneously differentially expressed in the tumor samples of the GSE33630 and GSE65144 datasets. Based on the 15 upregulated and 40 downregulated genes, the ATC and normal samples were clearly classified into two groups. The error assignment of ATC_2 was possibly due to sample quality or tumor heterogeneity. RT-PCR analysis established that $V C A N$ and COL5A1 were significantly expressed in 10 tumor tissues, compared with that in adjacent normal tissues and that the mRNA expression level of KCNJ16 was lower in the 10 tumor tissues.

Notably, the pathway enrichment analysis revealed that three downregulated DEGs including TG, TPO and TSHR were significantly involved in the autoimmune thyroid disease pathway. Although the relationship between thyroid cancer and autoimmune thyroid disease is unclear, the co-existance of these two clinical afflictions has been demonstrated by retro- 


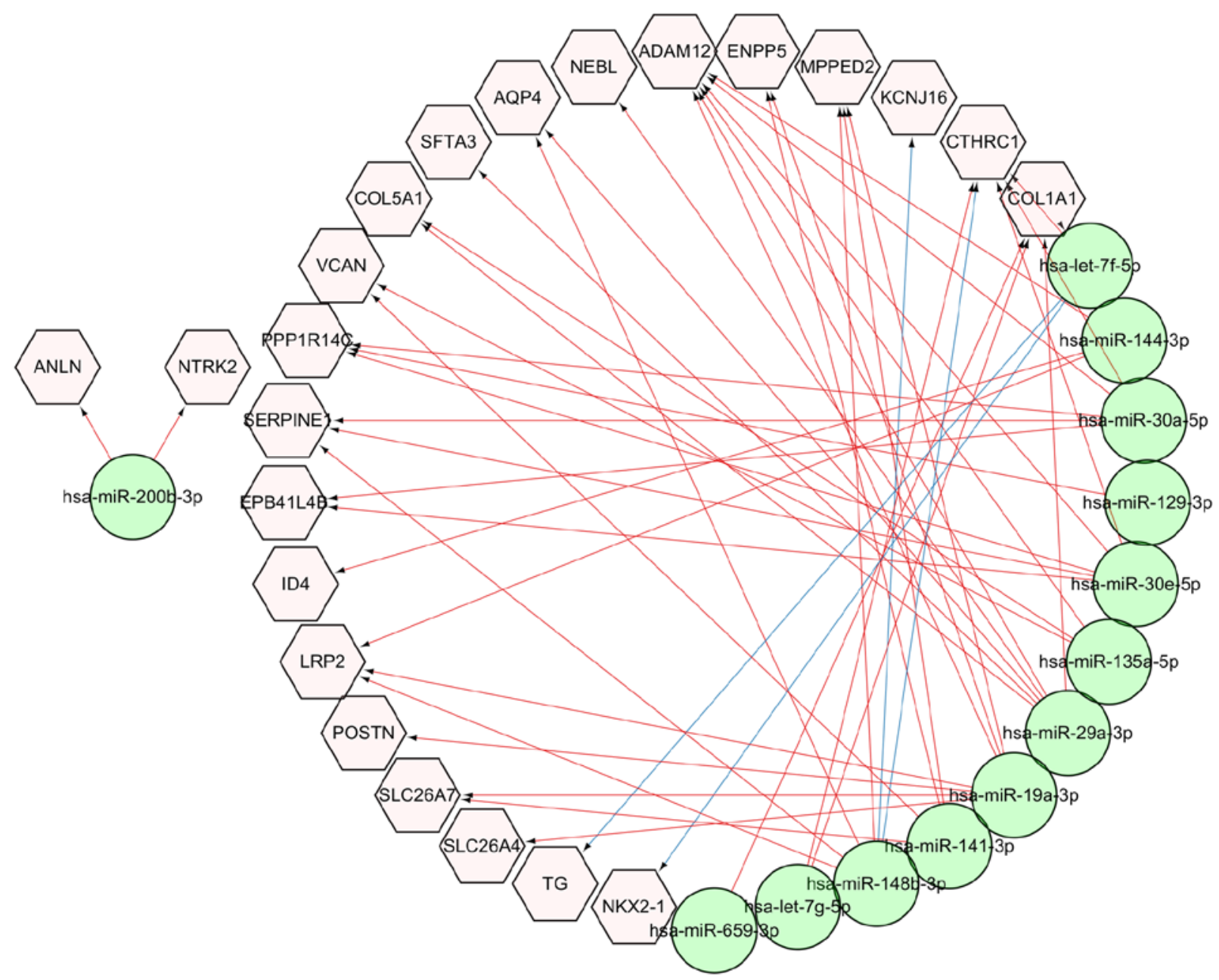

Figure 6. miRNA and mRNA interaction network from the miRTarBase (version 4.4) and the TargetScan database. The pink hexagons indicate the target genes predicted using the miRTarBase and the green circles indicate the miRNAs.

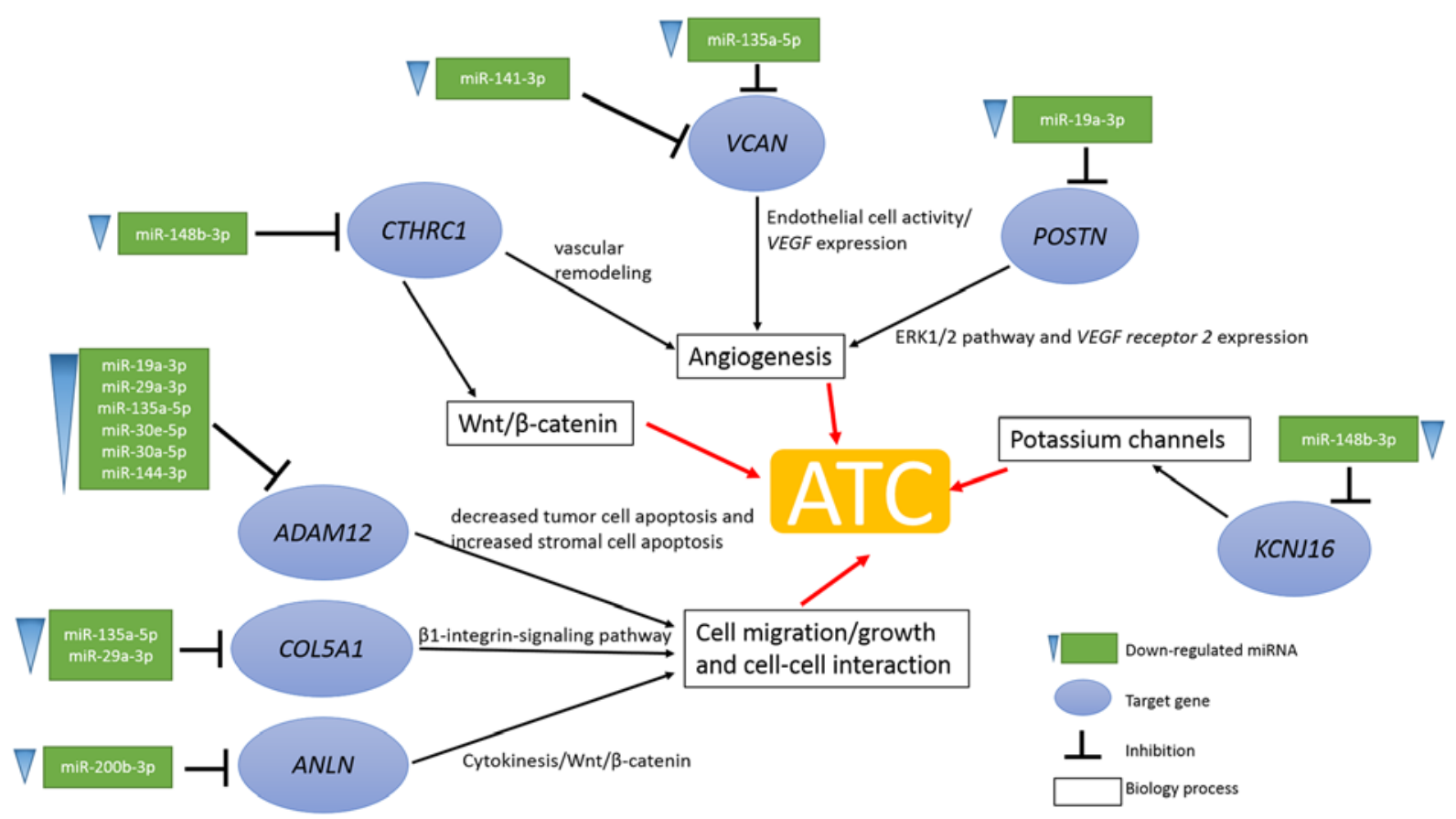

Figure 7. Possible molecular mechanism diagram of anaplastic thyroid carcinoma (ATC) based on the analysis results. The inverted triangles associated with the rectangles represent downregulated miRNA; ellipses represent target genes. Combined vertical and horizontal symbols represent inhibition. Rectangles represent biological processes. 
spective cohort analysis. One study showed that thyroid cancer is significantly associated with an elevated concentration of $\mathrm{TgAb}$ (thyroglobulin antibodies, $\mathrm{OR}=1.57 ; \mathrm{CI}=1.11-2.23$ ) based on the study of 253 patients with thyroid cancer (20). Moreover, one review systematically summarized the reports concerning the link between thyroid autoimmunity and differentiated thyroid cancer (DTC) (2). The low expression of $T G, T P O$ and $T S H R$ in this study was possibly caused by the lower expression of $N K X 2-1$ which can bind to thyroglobulin promoter and regulate thyroid functional gene expression (21). Research has shown that promoter hypermethylation of TSHR is significantly related to TSHR gene silencing (22). With relatively rich $\mathrm{CpG}$ dinucleotides, TSHR can be methylated (23) and suppress thyroid iodide-metabolizing molecules. Hence thyroid tumor cells are unable to concentrate iodine and are insensitive to radioiodine (24). These downregulated genes are possibly the results of thyroid cancer $(25,26)$.

Considering the complexity of cancer, the molecular mechanisms of ATC are far from clear. Based on the integration analysis of mRNA and miRNA expression (5), the unknown mechanisms of ATC were further revealed. Integrative analysis results showed that the progression of ATC is considered to be a multipath process including the angiogenesis process, the Wnt/ $\beta$-catenin pathway, cell migration or cell-cell interaction and potassium channel function (Fig. 7).

Research has shown that angiogenesis is critical for cancer cell proliferation and metastatic spread due to the requirement of tumors for adequate oxygen and nutrient supply (27). In the present study, the angiogenesis process was activated by the upregulated expression of CTHRC1, VCAN and POSTN and based on the miRNA regulation analysis results. The upregulated expression of CTHRC1, VCAN and POSTN was the consequence of the downregulated expression of miR-148b-3p, miR-141-3p, miR-135a-5p and miR-19a-3p, respectively (Fig. 7). Additionally, somatic mutation of $V C A N$ (D748G, missense) is possibly related to its functional upregulation (7). CTHRC1, as a novel oncogene, has been proved to be abnormally overexpressed in malignant tumors such as melanoma, breast, pancreas, human non-small cell lung and thyroid cancers (28-32). Overexpression of CTHRCl may contribute to vascular remodeling and cell migration by suppressing collage matrix deposition (33). Moreover, reports have shown that CTHRC1 anchoring on the cell membrane may stabilize the physical interaction between frizzled receptors and Wnt ligands, and activate the non-canonical Wnt pathway regulating cell motility (34). Moreover, $V C A N$, as a member of the versican proteoglycan family, is also valuable for angiogenesis. Yang and Yee reported that VCAN-transfected tumor cells, exhibited enriched vascularization and accumulation of red blood cells by H\&E staining compared with vector-transfected cells (35). In addition, the endothelial marker of blood vessel formation CD34 was significantly overexpressed in tumor sections (35). Immunocytochemistry analysis also showed that $V C A N$-transfected cells contained more and larger blood vessels than the control cells (36). These angiogenesis-related processes participated in the enhancement of endothelial cell activities and fibronectin and vascular endothelial growth factor (VEGF) expression (36). Furthermore, POSTN, an adhesion molecule in osteoblasts, was identified to be overexpressed and be related to the angiogenesis process. Research has been carried out to explore the relationship between POSTN and angiogenesis. In keloids, overexpression of POSTN was found to promote angiogenesis by inducing ERK1/2 and focal adhesion kinase pathways and by upregulating expression of $V E G F$ and angiopoietin-1 (37). Additionally, the upregulation of $V E G F$ receptor 2 was identified in human breast cancer with acquired POSTN expression (38), and POSTN promoted angiogenesis via the paracrine pathway by interacting in a $\alpha_{\mathrm{V}} \beta_{3^{-}}$and $\alpha_{\mathrm{V}} \beta_{5}$-dependent process in ovarian cancer (39). All in all, these three overexpressed genes possibly enhance ATC metastasis and progression via the angiogenesis process.

Moreover, cell migration, cell growth and cell-cell interactions are also critical in the development or metastasis of tumors. In the present study, ADAM12,COL5A1 and ANLN were identified to be overexpressed and their upregulation was possibly caused by the low expression of corresponding miRNAs such as miR-19a-3p, miR-29a-3p, miR-135a-5p, miR-30e-5p, miR-30a-5p, miR-144-3p, miR-135a-5p, miR-29a-3p and miR-200b-3p. Additionally somatic mutations of ANLN (R1095W, missense) and COL5A1 (G1348A, missense) were found to contribute to its upregulation (40). $A D A M 12$, as one of the disintegrins and metalloproteases, has been demonstrated to be involved in several pathological processes. Microarray experiments indicate that $A D A M 12$ is upregulated in aggressive fibromatosis. The mechanism of $A D A M 12$ in breast tumor has been systematically investigated and the results showed that $A D A M 12$ reduced tumor cell apoptosis and simultaneously increased stromal cell apoptosis (41). In serous ovarian carcinoma, high levels of ADAM12 mRNA were detected possibly caused by $T G F \beta$ signaling (42). Moreover, COL5Al encoding an $\alpha$-chain of fibrillar collagens also regulated cell migration and motility $(43,44)$. COL5A1 binds to $\alpha 2 \beta 1$-integrin or $\beta 1$-integrin receptor and activates the corresponding signaling pathways. In pancreatic ductal adenocarcinoma, high expression of COL5A 1 significantly affected cell adhesion, migration and viability based on results from $\beta 1$-integrin inhibition, siRNA ablation of COL5A1 expression and COL5A1 knockdown experiments (45). In regards to colorectal carcinogenesis, RT-PCR results showed that COL5A1 was co-expressed with COL11A1 in tumor samples rather than in normal samples (46). In addition, the actinbinding protein $A N L N$ is also critical for cell growth, migration and cytokinesis (47). Zhou et al showed that knockdown of ANLN markedly inhibited breast cancer cell line proliferation and colony formation, and more cells were blocked at the $\mathrm{G}_{2} / \mathrm{M}$ phase (47). Alhough a detailed mechanism of ANLN in carcinoma progression is still unclear, in silico pathway prediction indicated that the $\mathrm{Wnt} / \beta$-catenin signaling pathway is associated with $A N L N$ downstream regulation (48).

Moreover, ion channels especially potassium channels have been demonstrated to play a crucial role in tumors (49). In the present study, KCNJ16 was downregulated which led to an ion concentration unbalance between the extracellular and intracellular compartments. Rather than a single mechanism, potassium channel regulation may influence tumor progression via multiple paths such as cell adhesion or migration, angiogenesis and apoptosis (49). The complex regulation mechanism of KCNJ16 in ATC progression still remains to be explored.

In summary, the poor prognosis of ATC is possibly induced by various processes. Firstly, upregulation of CTHRC1, VCAN 
and $P O S T N$ promotes angiogenesis and provides necessary nutrition for tumor cells. Then ADAM12, COL5A1 and ANLN induce cell migration, cell growth or cell-cell interaction leading to tumor distant metastasis. Finally, $K C N J 16$ regulates intracellular and extracellular ion concentrations and promotes ATC progression.

\section{Acknowledgements}

This study was funded by the National Natural Science Foundation of Shanghai (12ZR1438700).

\section{References}

1. Nguyen QT, Lee EJ, Huang MG, Park YI, Khullar A and Plodkowski RA: Diagnosis and treatment of patients with thyroid cancer. Am Health Drug Benefits 8: 30-40, 2015.

2. Feldt-Rasmussen U and Rasmussen AK: Autoimmunity in differentiated thyroid cancer: Significance and related clinical problems. Hormones (Athens) 9: 109-117, 2010.

3. Carling T and Udelsman R: Thyroid cancer. Annu Rev Med 65: $125-137,2014$.

4. Smallridge RC, Marlow LA and Copland JA: Anaplastic thyroid cancer: Molecular pathogenesis and emerging therapies. Endocr Relat Cancer 16: 17-44, 2009.

5. Hébrant A, Floor S, Saiselet M, Antoniou A, Desbuleux A, Snyers B, La C, de Saint Aubain N, Leteurtre E, Andry G, et al: miRNA expression in anaplastic thyroid carcinomas. PLoS One 9: e103871, 2014

6. Hébrant A, Dom G, Dewaele M, Andry G, Trésallet C, Leteurtre E, Dumont JE and Maenhaut C: mRNA expression in papillary and anaplastic thyroid carcinoma: Molecular anatomy of a killing switch. PLoS One 7: e37807, 2012.

7. Kunstman JW, Juhlin CC, Goh G, Brown TC, Stenman A, Healy JM, Rubinstein JC, Choi M, Kiss N, Nelson-Williams C, et al: Characterization of the mutational landscape of anaplastic thyroid cancer via whole-exome sequencing. Hum Mol Genet 24: 2318-2329, 2015.

8. Wilkens L, Benten D, Tchinda J, Brabant G, Pötter E, Dralle H and von Wasielewski R: Aberrations of chromosomes 5 and 8 as recurrent cytogenetic events in anaplastic carcinoma of the thyroid as detected by fluorescence in situ hybridisation and comparative genomic hybridisation. Virchows Arch 436: 312-318, 2000.

9. von Roemeling CA, Marlow LA, Pinkerton AB, Crist A, Miller J, Tun HW, Smallridge RC and Copland JA: Aberrant lipid metabolism in anaplastic thyroid carcinoma reveals stearoyl CoA desaturase 1 as a novel therapeutic target. J Clin Endocrinol Metab 100: E697-E709, 2015.

10. Berger B, Peng J and Singh M: Computational solutions for omics data. Nat Rev Genet 14: 333-346, 2013.

11. Dom G, Tarabichi M, Unger K, Thomas G, OczkoWojciechowska M, Bogdanova T, Jarzab B, Dumont JE, Detours V and Maenhaut C: A gene expression signature distinguishes normal tissues of sporadic and radiation-induced papillary thyroid carcinomas. Br J Cancer 107: 994-1000, 2012.

12. Gentleman RC, Carey VJ, Bates DM, Bolstad B, Dettling M, Dudoit S, Ellis B, Gautier L, Ge Y, Gentry J, et al: Bioconductor: Open software development for computational biology and bioinformatics. Genome Biol 5: R80, 2004.

13. Kerr MK: Linear models for microarray data analysis: Hidden similarities and differences. J Comput Biol 10: 891-901, 2003.

14. Dennis G Jr, Sherman BT, Hosack DA, Yang J, Gao W, Lane HC and Lempicki RA: DAVID: Database for Annotation, Visualization, and Integrated Discovery. Genome Biol 4: 3, 2003.

15. Harris MA, Clark J, Ireland A, Lomax J, Ashburner M, Foulger R, Eilbeck K, Lewis S, Marshall B, Mungall C, et al; Gene Ontology Consortium: The Gene Ontology (GO) database and informatics resource. Nucleic Acids Res 32: D258-D261, 2004.

16. Kanehisa M and Goto S: KEGG: Kyoto encyclopedia of genes and genomes. Nucleic Acids Res 28: 27-30, 2000.

17. Warde-Farley D, Donaldson SL, Comes O,Zuberi K, Badrawi R, Chao P, Franz M, Grouios C, Kazi F, Lopes CT, et al: The GeneMANIA prediction server: Biological network integration for gene prioritization and predicting gene function. Nucleic Acids Res 38: W214-W220, 2010.
18. Kutmon M, Kelder T, Mandaviya P, Evelo CT and Coort SL: CyTargetLinker: A cytoscape app to integrate regulatory interactions in network analysis. PLoS One 8: e82160, 2013.

19. Hsu SD, Lin FM, Wu WY, Liang C, Huang WC, Chan WL, Tsai WT, Chen GZ, Lee CJ, Chiu CM, et al: miRTarBase: A database curates experimentally validated microRNA-target interactions. Nucleic Acids Res 39: D163-D169, 2011.

20. Azizi G and Malchoff CD: Autoimmune thyroid disease: A risk factor for thyroid cancer. Endocr Pract 17: 201-209, 2011.

21. Ma R, Latif R and Davies TF: Thyroid follicle formation and thyroglobulin expression in multipotent endodermal stem cells. Thyroid 23: 385-391, 2013.

22. Khan MS, Pandith AA, Masoodi SR, Wani KA, Ul Hussain M and Mudassar S: Epigenetic silencing of TSHR gene in thyroid cancer patients in relation to their BRAF V600E mutation status. Endocrine 47: 449-455, 2014.

23. Xing M, Usadel H, Cohen Y, Tokumaru Y, Guo Z, Westra WB, Tong BC, Tallini G, Udelsman R, Califano JA, et al: Methylation of the thyroid-stimulating hormone receptor gene in epithelial thyroid tumors: A marker of malignancy and a cause of gene silencing. Cancer Res 63: 2316-2321, 2003.

24. Smith JA, Fan CY, Zou C, Bodenner D and Kokoska MS: Methylation status of genes in papillary thyroid carcinoma. Arch Otolaryngol Head Neck Surg 133: 1006-1011, 2007.

25. Kowalska A, Pałyga I, Gąsior-Perczak D, Walczyk A, Trybek T, Słuszniak A, Mężyk R and Góźdź S: The cut-off level of recombinant human TSH-stimulated thyroglobulin in the follow-up of patients with differentiated thyroid cancer. PLoS One 10: e0133852, 2015.

26. Teama SH, Agwa SH, Fawzy A, Sayed MM, Ibrahim WA and Eid YM: Molecular detection of circulating thyroid specific transcripts (TSHR/Tg-mRNAs) in thyroid cancer patients: Their diagnostic significance. Egypt J Med Hum Genet 12: 201-209, 2011.

27. Nishida N, Yano H, Nishida T, Kamura T and Kojiro M: Angiogenesis in cancer. Vasc Health Risk Manag 2: 213-219, 2006.

28. Ip W, Wellman-Labadie O, Tang L, Su M, Yu R, Dutz J, Wang Y, Huang S, Zhang X, Huang C, et al: Collagen triple helix repeat containing 1 promotes melanoma cell adhesion and survival. J Cutan Med Surg 15: 103-110, 2011.

29. LeClair R and Lindner V: The role of collagen triple helix repeat containing 1 in injured arteries, collagen expression, and transforming growth factor beta signaling. Trends Cardiovasc Med 17: 202-205, 2007.

30. Durmus T, LeClair RJ, Park KS, Terzic A, Yoon JK and Lindner V: Expression analysis of the novel gene collagen triple helix repeat containing-1 (Cthrc1). Gene Expr Patterns 6: 935-940, 2006.

31. Tang L, Dai DL, Su M, Martinka M, Li G and Zhou Y: Aberrant expression of collagen triple helix repeat containing 1 in human solid cancers. Clin Cancer Res 12: 3716-3722, 2006.

32. Turashvili G, Bouchal J, Ehrmann J, Fridman E, Skarda J and Kolar Z: Novel immunohistochemical markers for the differentiation of lobular and ductal invasive breast carcinomas. Biomed Pap Med Fac Univ Palacky Olomouc Czech Repub 151: 59-64, 2007.

33. Pyagay P, Heroult M, Wang Q, Lehnert W, Belden J, Liaw L, Friesel RE and Lindner V: Collagen triple helix repeat containing 1, a novel secreted protein in injured and diseased arteries, inhibits collagen expression and promotes cell migration. Circ Res 96: 261-268, 2005.

34. Ke Z, He W, Lai Y, Guo X, Chen S, Li S, Wang Y and Wang L: Overexpression of collagen triple helix repeat containing 1 (CTHRC1) is associated with tumour aggressiveness and poor prognosis in human non-small cell lung cancer. Oncotarget 5: 9410-9424, 2014.

35. Yang W and Yee AJ: Versican V2 isoform enhances angiogenesis by regulating endothelial cell activities and fibronectin expression. FEBS Lett 587: 185-192, 2013.

36. Zheng PS, Wen J, Ang LC, Sheng W, Viloria-Petit A, Wang Y, $\mathrm{Wu}$ Y, Kerbel RS and Yang BB: Versican/PG-M G3 domain promotes tumor growth and angiogenesis. FASEB J 18: 754-756, 2004.

37. Zhang Z, Nie F, Chen X, Qin Z, Kang C, Chen B, Ma J, Pan B and Ma Y: Upregulated periostin promotes angiogenesis in keloids through activation of the ERK 1/2 and focal adhesion kinase pathways, as well as the upregulated expression of VEGF and angiopoietin 1. Mol Med Rep 11: 857-864, 2015. 
38. Shao R, Bao S, Bai X, Blanchette C, Anderson RM, Dang T, Gishizky ML, Marks JR and Wang XF: Acquired expression of periostin by human breast cancers promotes tumor angiogenesis through up-regulation of vascular endothelial growth factor receptor 2 expression. Mol Cell Biol 24: 3992-4003, 2004.

39. Zhu M, Fejzo MS, Anderson L, Dering J, Ginther C, Ramos L, Gasson JC, Karlan BY and Slamon DJ: Periostin promotes ovarian cancer angiogenesis and metastasis. Gynecol Oncol 119: 337-344, 2010

40. Kunstman JW, Juhlin CC, Goh G, Brown TC, Stenman A, Healy JM, Rubinstein JC, Choi M, Kiss N, Nelson-Williams C, et al: Characterization of the mutational landscape of anaplastic thyroid cancer via whole-exome sequencing. Hum Mol Genet 24 2318-2329, 2015.

41. Kveiborg M, Fröhlich C, Albrechtsen R, Tischler V, Dietrich N, Holck P, Kronqvist P, Rank F, Mercurio AM and Wewer UM: A role for ADAM12 in breast tumor progression and stromal cell apoptosis. Cancer Res 65: 4754-4761, 2005.

42. Cheon DJ, Li AJ, Beach JA, Walts AE, Tran H, Lester J, Karlan BY and Orsulic S: ADAM12 is a prognostic factor associated with an aggressive molecular subtype of high-grade serous ovarian carcinoma. Carcinogenesis 36: 739-747, 2015.

43. Larsen M, Tremblay ML and Yamada KM: Phosphatases in cell-matrix adhesion and migration. Nat Rev Mol Cell Biol 4: $700-711,2003$
44. Murasawa Y, Hayashi T and Wang P-C: The role of type V collagen fibril as an ECM that induces the motility of glomerular endothelial cells. Exp Cell Res 314: 3638-3653, 2008.

45. Berchtold S, Grünwald B, Krüger A, Reithmeier A, Hähl T, Cheng T, Feuchtinger A, Born D, Erkan M, Kleeff J, et al: Collagen type $\mathrm{V}$ promotes the malignant phenotype of pancreatic ductal adenocarcinoma. Cancer Lett 356: 721-732, 2015.

46. Fischer H, Stenling R, Rubio C and Lindblom A: Colorectal carcinogenesis is associated with stromal expression of COL11 A1 and COL5A2. Carcinogenesis 22: 875-878, 2001.

47. Zhou W, Wang Z, Shen N, Pi W, Jiang W, Huang J, Hu Y, Li X and Sun L: Knockdown of ANLN by lentivirus inhibits cell growth and migration in human breast cancer. Mol Cell Biochem 398: $11-19,2015$

48. Pandi NS, Manimuthu M, Harunipriya P, Murugesan M, Asha GV and Rajendran S: In silico analysis of expression pattern of a Wnt $/ \beta$-catenin responsive gene ANLN in gastric cancer. Gene 545: 23-29, 2014.

49. Pardo LA and Stühmer W: The roles of $\mathrm{K}(+)$ channels in cancer. Nat Rev Cancer 14: 39-48, 2014 